\title{
Intercellular Communication between Malaria-Infected Erythrocytes in the Accelerated Multidrug Resistance Phenotype of the Plasmodium falciparum Parasite
}

\author{
Olaitan O Omitola ${ }^{1}$ and Andrew W Taylor-Robinson ${ }^{2^{*}}$
}

${ }^{1}$ Department of Pure and Applied Zoology, Federal University of Agriculture, Abeokuta, Nigeria

${ }^{2}$ Infectious Diseases Research Group, School of Medical and Applied Sciences, Central Queensland University, Rockhampton, Australia

\begin{abstract}
The so-called ARMD ('accelerated resistance to multidrug') phenotype of the human malaria parasite Plasmodium falciparum that is prevalent in South East Asia is believed to confer a special hypermutator characteristic upon parasite strains in which it is expressed. Examination of the ARMD phenotype suggests that alternative mechanisms may be responsible for this ability that is unique among strains of $P$. falciparum parasite worldwide, but over which there is concern that further dissemination may lead to widespread loss of sensitivity to current chemotherapeutic regimens. Therefore, other recently discovered mechanisms that demonstrate potential in contributing to this noted drug resistance phenomenon merit further investigation. One important example, the cell-to-cell communication pathway in $P$. falciparum-infected erythrocytes, is being examined as a potential target for intervention and forms the subject of this article.
\end{abstract}

Keywords: Malaria; Plasmodium falciparum; Chemotherapy; Drug resistance; Phenotype; ARMD; Hypermutator

\section{Introduction}

Malaria in humans is caused by five species of protozoan parasite of the genus Plasmodium that are transmitted by Anopheles mosquitoes $[1,2]$. The disease is endemic to each non-polar continent but occurs predominantly in tropical regions $[3,4]$. Of the current estimated global population of 7.3 billion people [5], around 1.5 billion are infected with malaria parasites and 3.4-3.7 billion are at risk of contracting malaria $[4,6]$. It is claimed routinely that there are 300-500 million clinical cases worldwide annually [7]. The 1-3 million deaths that result equate to a person dying every $10-30$ seconds [6]. $90 \%$ of deaths are due to $P$. falciparum [8]. Lethality is associated with increased risk of parasite resistance developing to current anti-malarial drugs [9].

\section{Anti-Malarial Drug Resistance and South East Asia}

Drug resistance by the pathogenic erythrocyte-infecting stages of P. falciparum is evidently a continuing major issue among the global health community and in those areas where it develops this is often accompanied by an upsurge in reporting of cases of morbidity and mortality [10]. P. falciparum has evolved serial loss of sensitivity to several prophylactic drugs such as chloroquine and sulfadoxinepyrimethamine used heavily in chemotherapeutic treatment of the infection [11]. Alarmingly, the parasite developed resistance to atovaquone in the same year it was introduced and also to other initially effective drugs such as mefloquine [12]. Most recently, concern has followed reports of emerging resistance to the current frontline chemotherapeutic anti-malarial drug, artemisinin, arising in South East Asia [13], with the real potential of this spreading to high-risk endemic regions like sub-Saharan Africa where mutations in the K13-propeller genetic marker are already noted in some locations [14].

An intriguing aspect for malaria specialists is that the history of resistance to drugs exhibited by $P$. falciparum shows a relatively consistent pattern of emergence and subsequent spread from South East Asia to the rest of the world [15]. Resistance to mefloquine was reported to have occurred within six years of systematic deployment confined mainly to Thailand, Cambodia and Vietnam [16]. The chloroquine resistance that is now prevalent in Africa is believed to have arisen by importation from Asia [17,18], while the first confirmed cases of a decline in efficacy of artemisinin treatment came from Cambodia $[12,13,19]$.

Several studies have attempted to link the rapid emergence of $P$. falciparum resistance to anti-malarials in South East Asia with various factors such as monotherapeutic treatments, black marketing of subtherapeutic dosages, extensive drug misuse, non-immune migrants $[11,12,20]$, but these do not explain sufficiently the association of this area with the origins of drug resistance and dissemination to other regions. Drug failures in carefully controlled clinical settings were reported in Thailand [20], and artemisinin resistance is said to have emerged independently on at least three different occasions in South East Asia [21]. Indeed, the local strain of $P$. falciparum possesses an exceptional capacity to evolve resistance to drugs more rapidly than do other strains $[11,20,22]$. It is this remarkable characteristic that has been identified as the ARMD ('accelerated resistance to multidrug') phenotype [20]. This has shown significant phenotypic variation in vitro between geographically distinct populations of the parasite, the South East Asian strain exhibiting about 1,000-fold stronger tendency to evolve drug resistance than the African strain [20].

\section{The Hypermutator ARMD Hypothesis}

Following in vitro demonstration of the ARMD phenotype [20], it was generally believed that a hypermutator trait accounts for this observed characteristic of the South East Asian P. falciparum strain compared to non-South East Asian strainsand which enables acquisition

*Corresponding author: AW Taylor-Robinson, School of Medical \& Applied Sciences, Central Queensland University, Rockhampton, QLD 4702, Australia, Tel: +61 74923 2008; E-mail: a.taylor-robinson@cqu.edu.au

Received: March 17, 2016; Accepted: June 08, 2016; Published: June 15, 2016

Citation: Omitola OO, Taylor-Robinson AW (2016) Intercellular Communication between Malaria-Infected Erythrocytes in the Accelerated Multidrug Resistance Phenotype of the Plasmodium falciparum Parasite. J Infect Dis Med 1: 105. doi: 10.4172/2576-1420.1000101

Copyright: (C) 2016 Omitola OO, et al. This is an open-access article distributed under the terms of the Creative Commons Attribution License, which permits unrestricted use, distribution, and reproduction in any medium, provided the original author and source are credited. 
of single nucleotide polymorphisms (SNPs) and new drug resistance traits at an accelerated rate $[18,21]$. A mutator phenotype likened to that observed in some bacteria and yeast may account for this increased propensity for mutation in the ARMD P. falciparum phenotype [20]. This hypothetical mechanism is apparently supported by the unusual population structure and high level of genetic differentiation observed in P. falciparum samples from Cambodia where several subpopulations associated with clinical artemisinin-resistance exhibit high frequencies of SNPs [21].

It should be stressed that the hypermutator trait is a rare phenomenon among eukaryotic parasites, unlike in eubacterial pathogens, and has not knowingly been observed ex vivo in the phylum Apicomplexa of protozoan parasites that includes Plasmodium [23]. While an investigation published earlier this year suggests that a mild mutator phenotype may exist in the South East Asian parasite population [18], in silico analysis of $P$. falciparum field isolates from geographically distant countries like Mali and Bangladesh revealed contraindicatory evidence of significantly higher substitution rates [23]. This research was the first attempt to study the ARMD phenomenon using field isolates of $P$. falciparum.

Notwithstanding the demonstration of a mild mutator phenotype in the South East Asian strain [18], drug resistance mutations are thought to be spontaneous genetic events in P. falciparum [11]. Thus, mutations rates may not vary significantly among strains or play a determinant role in the exceptional ability to evolve loss of drug sensitivity. This notion may be corroborated by showing that the extent of mutations appearing in different South East Asian and non-South East Asian strains of P. falciparum is not associated significantly with an increased predisposition to develop drug resistance $[18,23]$. This implies that the ARMD phenotype may not be derived from a higher level of mutation frequencies in South East Asian isolates, as might have been expected previously.

\section{Alternative Explanations of the ARMD Mechanism}

The propensity of $P$. falciparum to establish resistance successfully to novel therapeutic drugs depends, in addition to the mutant drug resistance gene, on its ability to develop subsequent compensatory mutation(s) for fitness [22,24]. It is assumed that $P$. falciparum parasites carrying drug resistance mutations which are associated with a significant fitness cost are more likely to be outcompeted by drugsensitive but otherwise fitter parasites and thus, that such resistance will not be transmitted efficiently in the population to become observable clinically [22]. Hence, pressure is imposed on P. falciparum to enhance the process of selection of compensatory mutations, which is widely believed to be achieved through high parasitemias during infection or influenced by other factors from the human host such as strong drug pressure and drug misuse [22,25].

The implication is that the South East Asia strain of $P$. falciparum has been able to develop a more enhanced ability for selection of drug resistance and compensatory mutations. It may be implied further that while drug resistance mutations can evolve spontaneously in all strains at similar rates, the protective effect will not be observable clinically/phenotypically in a parasite strain until resistant mutants are sufficiently fit so that loss of drug sensitivity can be transmitted at a significant level within the population [11]. Therefore, the unique ARMD phenotype for rapid development of clinically observable drug resistance may not in fact be associated with the strain's ability to demonstrate a higher frequency of mutations than that observed in other isolates per se. Rather, it may be linked to the ability to employ efficiently mechanisms that facilitate the acquisition of compensatory mutations which specifically favour fitness and concentrate them in 'unfit' resistant mutants before they are outcompeted, thereby achieving drug resistance fitness and clinical manifestations faster than other strains when these 'fit' mutations are transmitted within the $P$. falciparum population. Such inherent traits in $P$. falciparum strains may not be detectable by measuring the frequency of mutations that appear in the overall parasite population.

It has been suggested previously that meiotic recombination may assist the concentration of independently acquired resistance traits into single clones [20], which may explain the mechanism underlying the ARMD phenomenon. However, in experimental animal models drug resistance mutations can be selected for without meiotic recombination $[11,26]$. Indeed, frequent genetic recombination due to high levels of transmission occurs in Africa, where the ARMD phenotype is currently weakest [27]. This trait apparently still remains strongest in the South East Asia strain of $P$. falciparum, as was exhibited recently in the emerging resistance to artemisinin that originated in Cambodia [13].

This finding shows that, unlike for meiotic recombination, those mechanisms which enhance noticeably the fitness of drug resistance mutations and contribute to the ARMD phenotype will demonstrate a measure of less random selection and more precision in transmitting genes that promote survival of $P$. falciparum from prevailing drug pressure. In addition to its tendency to be amplified significantly by high parasitemia, as was similarly observed in the ARMD phenotype [20], such precise and selective transmission of genes is demonstrated by the cell-to-cell communication mechanism utilized in P. falciparum [28]. This previously unknown phenomenon of intercellular exchange in malaria parasites was reported recently to occur in vitro between $P$. falciparum-infected erythrocytes through the production of exosome-like vesicles. This showed an involvement in signaling of gametocytogenesis but was also implicated in transfer of DNA materials relating to conferral of drug resistance [29,30], hence promoting resistance in previously drug-sensitive parasites [28].

\section{Intercellular Communication and the ARMD Phenotype}

The process described here may also serve as a potential mechanism to facilitate the pathway to fitness of novel drug resistance mutations in P. falciparum, whereby parasites are able to cross-transfer both drug resistance genes and advantageous compensatory mutations selected under drug pressure. Subsequently, such parasites would be able to develop significant clinical resistance faster than by depending solely on high parasitemia or high mutation frequencies for selection of compensatory mutations. It was hitherto thought that the South East Asian $P$. falciparum strain may employ enhanced recombinationbased strategies that facilitate frequent changes in gene expression or loss of function mutation [20], thus assisting development of drug resistance. Therefore, the cell-to-cell communication mechanism may also provide a possible pivotal function in the ARMD phenotype such that $P$. falciparum parasites bearing mutant resistance genes and under both drug pressure and fitness stress are able to acquire fitness genes more rapidly by horizontal transfer of favourable compensatory genes through more efficient use of intercellular communication than shown by other strains. Indeed, drug stress appears to influence heightened resistance gene transfer between P. falciparum parasites [28]; hence, fitness stress may also play a determinant role in gene transfer via intercellular communication.

\section{Future Research Directions}

In order to show that intercellular communication in the malaria 
Citation: Omitola OO, Taylor-Robinson AW (2016) Intercellular Communication between Malaria-Infected Erythrocytes in the Accelerated Multidrug Resistance Phenotype of the Plasmodium falciparum Parasite. J Infect Dis Med 1: 105. doi: 10.4172/2576-1420.1000101

parasite is not only a potential mechanism for spread of already established drug resistance but to determine if it also facilitates development of novel resistance, further research is required. The focus of investigation should be the putative functional role of $P$. falciparum parasite intercellular communication in the ARMD phenotype by examining for horizontal transfer of compensatory mutation genes along with drug resistance genes between parasites in the presence of drug pressure and fitness stress. Based on the established variability of cell-to-cell communication efficiency at different developmental stages of the parasite, the possibility of variant efficiency of intercellular communication among $P$. falciparum strains should also be explored as this may explain how the ARMD phenotype is exceptionally stronger in the South East Asia strain than in any other P. falciparum parasite clone.

\section{Conclusion}

In the almost two decades since its first recognition [20], the crucial role in malaria parasite drug resistance which is played by the ARMD phenotype that bestows a unique hypermutator trait on isolates of $P$. falciparum is becoming increasingly apparent. Efforts have been made to dissect the underlying mechanism(s) in the expectation that this may reveal targets for intervention. Among these, the role of intercellular communication in P. falciparum-infected erythrocytes is being studied in vitro by comparing cell-to-cell communication efficiencies across parasite clones displaying different ARMD phenotypes and subjected to anti-malarial drug pressure. This may shed light on the possible contribution of intercellular communication to multidrug resistance in the major human malaria parasite.

\section{Conflict of Interests}

The authors do not have any conflict of interest to declare.

\section{References}

1. Moya A, Font E (2004) Evolution: From Molecules to Ecosystems. Oxford University Press, Oxford.

2. Singh B, Kim Sung L, Matusop A, Radhakrishnan A, Shamsul SS, et al. (2004) A large focus of naturally acquired Plasmodium knowlesi infections in human beings. Lancet 363: 1017-1024.

3. Centers for Disease Control and Prevention (2016) Where Malaria Occurs.

4. Breman JG (2009) Eradicating malaria. Sci Prog 92: 1-38.

5. U.S. Census Bureau (2016) World Population Clock.

6. Hay SI, Guerra CA, Tatem AJ, Noor AM, Snow RW (2004) The global distribution and population at risk of malaria: past, present, and future. Lancet Infect Dis 4: 327-336.

7. Johns Hopkins Malaria Research Institute (2016) About Malaria

8. World Health Organization (2015) Malaria Fact Sheet.

9. Barik TK (2015) Antimalarial drug: from its development to deface. Curr Drug Discov Technol 12: 225-228.

10. World Health Organization (2015) World Malaria Report 2015. WHO Press, Geneva.

11. White NJ (2004) Antimalarial drug resistance. J Clin Invest 113: 1084-1092.
12. Winzeler EA, Manary MJ (2014) Drug resistance genomics of the antimalarial drug artemisinin. Genome Biol 15: 544.

13. Saunders DL, Vanachayangkul P, Lon C; U.S. Army Military Malaria Research Program; National Center for Parasitology, Entomology, and Malaria Control (CNM), et al. (2014) Dihydroartemisinin-piperaquine failure in Cambodia. N Engl J Med 371: 484-485.

14. Kamau E, Campino S, Amenga-Etego L, Drury E, Ishengoma D, et al. (2015) $\mathrm{K} 13-$ propeller polymorphisms in Plasmodium falciparum parasites from subSaharan Africa. J Infect Dis 211: 1352-1355

15. Ariey F, Witkowski B, Amaratunga C, Beghain J, Langlois AC, et al. (2014) A molecular marker of artemisinin-resistant Plasmodium falciparum malaria. Nature 505: 50-55.

16. Nosten F, van Vugt M, Price R, Luxemburger C, Thway KL, et al. (2000) Effects of artesunate-mefloquine combination on incidence of Plasmodium falciparum malaria and mefloquine resistance in western Thailand: a prospective study. Lancet 356: 297-302.

17. Wootton JC, Feng X, Ferdig MT, Cooper RA, Mu J, et al. (2002) Genetic diversity and chloroquine selective sweeps in Plasmodium falciparum. Nature 418: 320-323.

18. Lee AH, Fidock DA (2016) Evidence of a mild mutator phenotype in Cambodian Plasmodium falciparum malaria parasites. PLoS One 11: e0154166.

19. Tanabe K, Zakeri S, Palacpac NMQ, Afsharpad M, Randrianarivelojosia M, et al. (2011) Spontaneous mutations in the Plasmodium falciparum sarcoplasmic endoplasmic reticulum $\mathrm{Ca}^{2+}$-ATPase (PfATP6) gene among geographically widespread parasite populations unexposed to artemisinin-based combination therapies. Antimicrob Agents Chemother 55: 94-100.

20. Rathod PK, McErlean T, Lee PC (1997) Variations in frequencies of drug resistance in Plasmodium falciparum. Proc Natl Acad Sci U S A 94: 9389-9393.

21. Miotto O, Almagro-Garcia J, Manske M, Macinnis B, Campino S, et al. (2013) Multiple populations of artemisinin-resistant Plasmodium falciparum in Cambodia. Nat Genet 45: 648-655.

22. Petersen I, Eastman R, Lanzer M (2011) Drug-resistant malaria: molecular mechanisms and implications for public health. FEBS Lett 585: 1551-1562.

23. Brown TS, Silva JC, Djimdé A, Fukuda M, Kyaw MP, et al. (2015) Plasmodium falciparum field isolates from areas of repeated emergence of drug resistant malaria show no evidence of hypermutator phenotype. Infect Genet Evol 30: 318-322.

24. Levin BR, Perrot V, Walker N (2000) Compensatory mutations, antibiotic resistance and the population genetics of adaptive evolution in bacteria Genetics 154: 985-997.

25. Hastings IM, Paget-McNicol S, Saul A (2004) Can mutation and selection explain virulence in human P. falciparum infections? Malar J 3: 2.

26. Peters W (1987) Chemotherapy and Drug Resistance in Malaria (2nd edn) Academic Press, London.

27. Le Bras J, Durand R (2003) The mechanisms of resistance to antimalaria drugs in Plasmodium falciparum. Fundam Clin Pharmacol 17: 147-153.

28. Regev-Rudzki N, Wilson DW, Carvalho TG, Sisquella X, Coleman BM, et al. (2013) Cell-cell communication between malaria-infected red blood cells via exosome-like vesicles. Cell 153: 1120-1133

29. Lopez MA, Nguyen HT, Oberholzer M, Hill KL (2011) Social parasites. Curr Opin Microbiol 14: 642-648.

30. Rupp I, Sologub L, Williamson KC, Scheuermayer M, Reininger L, et al. (2011) Malaria parasites form filamentous cell-to-cell connections during reproduction in the mosquito midgut. Cell Res 21: 683-696. 